\title{
Nonlinear Model Predictive Control for Ecological Driver Assistance Systems in Electric Vehicles ${ }^{\text {th }}$
}

\author{
Seyed Amin Sajadi-Alamdari ${ }^{a, *}$, Holger $\operatorname{Voos}^{\mathrm{a}}$, Mohamed Darouach ${ }^{\mathrm{b}}$ \\ ${ }^{a}$ Interdisciplinary Centre for Security, Reliability and Trust (SnT), University of \\ Luxembourg, 29, avenue JF Kennedy, L-1855 Luxembourg \\ ${ }^{b}$ Centre de Recherche en Automatique de Nancy (CRAN) UMR-CNRS 7039, Université de \\ Lorraine, IUT de Longwy, 186 rue de Lorraine, F-54400 Cosnes et Romain, France
}

\begin{abstract}
Battery Electric Vehicle (BEV) has one of the most promising drivetrain technology. However, the BEVs are facing the limited cruising range which generally reduces their share in the automotive market. Velocity profile, acceleration characteristics, road gradients, and drive techniques around curves have significant impacts on the energy consumption of the BEVs. A semi-autonomous ecological driver assistance system to regulate the velocity with energy-efficient techniques is proposed to address the limitation. The main contribution of this paper is the design of a real-time nonlinear model predictive controller with improved inequality constraints handling and economic penalty function to plan the online cost-effective cruising velocity. This system is based on the extended cruise control driver assistance system which controls the longitudinal velocity of the $\mathrm{BEV}$ in a safe and energy efficient manner by taking advantage of road slopes, effective drive around curves, and respecting the traffic regulation. A realtime optimisation algorithm is adapted and extended with economic objective function. Instead of the conventional Euclidean norms, deadzone penalty functions are proposed to achieve the economic objectives. In addition, the states
\end{abstract}

\footnotetext{
This work is supported by the FNR "Fonds national de la Recherche" (Luxembourg) through the AFR" Aides à la Formation-Recherche" Ph.D. grant scheme No. 7041503.

* Corresponding author

Email addresses: amin.sajadi@uni.lu (Seyed Amin Sajadi-Alamdari), holger.voos@uni.lu (Holger Voos), mohamed.darouach@univ-lorraine.fr (Mohamed Darouach)
}

Preprint submitted to Journal of Robotics and Autonomous Systems

December 11, 2018 
inequality constraints are handled based on the proposed soft nonlinear complementarity function aimed to preserve the relaxed complementary slackness to enhance the Pontryagin's Minimum Principle (PMP) method. Obtained numerical simulation and field experimental results demonstrate the effectiveness of the proposed method for a semi-autonomous electric vehicle in terms of realtime energy-efficient velocity regulation and constraints satisfaction intended to improve the cruising range capability of the BEVs.

Keywords: Nonlinear Model Predictive Control; Ecological Driver Assistance Systems; Electric Vehicles; Optimal Energy Management; Real-Time Systems

\section{Introduction}

Advancement of vehicle technologies has improved their performance and the travel range tremendously. Modern technology of vehicles is also associated with its own challenges in safety, energy requirement, and environmental 5 impacts. A large number of Internal Combustion Engine (ICE) vehicles in use lead to serious problems for the environment and human life around the world such as air pollution and global warming [1. It is now well recognised that $\mathrm{BEVs}$ have one of the most promising powertrain technology for the predictable future transportations. The BEVs offer the same or even better performance in comparison to the ICE vehicles. Furthermore, the BEVs offer the opportunity to use different renewable energy resources.

The BEVs have limited onboard energy capacity, that limits their cruising range on a single charge, which is also known as range anxiety. Several investigations with the aim of elaborating performance of battery have been initiated $[2,3]$. However, most of these advances have failed to commercialise and are not foreseen to become available in the near future. In addition to battery research, current research and development remarkably focus on various ways of saving energy consumption that lead to extending the cruising range of the BEVs. Velocity profile, acceleration characteristics, road gradients, and drive techniques around curves have significant impacts on the energy consumption of the BEVs. 
The goal of Ecological (Eco) driving is to adapt the driving strategy to an energy-aware anticipative driving strategy. In realizing the energy-efficient driving, the driver has to demonstrate proper reactions to the anticipated traffic situations. However, drivers do not always and under all circumstances drive ecologically. Moreover, driver's Eco-driving mental workload is still high which may lead to their distraction [4]. The Ecological Driver Assistance Systems (EDAS) have high potentials to improve the safety and efficiency of the transportation network. Improving the efficiency by controlling the driving profile reveals its potential when considering that it does not require structural changes to the system [5].

Control technologies of the EDAS play an important role in robustness and performance of the system. There are multiple design objectives in the EDAS controller design which some of these objectives are contradictory. In addition, the EDAS controller has hard constraints such as actuators limit and soft constraints such as safety limits that need to be considered. Receding horizon optimal control also known as Model Predictive Control (MPC) has been an attractive approach for Mechatronics systems including the EDAS applications 6, 7, 8, 9. In the MPC, an Optimal Control Problem (OCP) is solved repeatedly in a receding horizon principle. The first element in the sequence of finite 40 control actions is applied to the system at each sampling time.

Ecological Cruise Control (Eco-CC) systems are one of the well-established EDAS applications that automate the throttle and brake control of the vehicle to retain the preset longitudinal velocity. Several works of literature may be founded, such as [10], where a predictive Eco-CC system was developed that minimises ICE vehicle fuel consumption levels utilising roadway topographic information. A comparison and assessments of fuel consumption models, cost functions, and solution methods on the fuel efficiency of ICE vehicles was presented in [11. An Eco-driving system for running a vehicle on roads with up-down slopes was developed in [12, which the fuel consumption model of a 50 typical ICE vehicle is formulated using engine efficiency characteristics and used in the objective function to ensure efficient fuel economy driving. 
The EDAS applications for the BEVs differ from the ones applied to the ICE vehicles due to specific design requirements of the BEVs [13]. For instance, the EDAS applications should consider the dynamics of the BEVs with the lower centre of gravity and its energy consumption characteristic map including the regenerative braking operating region. The Eco-CC applications for the $\mathrm{BEVs}$ received relatively little attention in works of literature. An energyefficient linear MPC that considers the energy consumption map of a BEV was established in 14, 15, 16, 17. Another anti-jerk model predictive cruise controller for electric vehicles adaptive to road conditions was proposed in [18. Nonlinear Model Predictive Control (NMPC) is distinguished by the use of nonlinear system models in the OCP to improve performance specifications [19]. A Nonlinear MPC (NMPC) problem formulation of an energy efficient Eco-CC system for electric vehicles was presented in [20]. Another NMPC based EcoCC system with extended functionalities to deal with hilly and curvy roads was proposed in 21].

Although most of the mentioned NMPCs are based on agile and intuitive set-point tracking, this may not be a suitable strategy for the energy-efficient state regulation. One of the main reason for high energy consumption of the system is strict achieving and tracking the set-point. Considering the general class of (residual) penalty functions used in the NMPC, the $\ell_{2}$-norm is preferred in practice due to its efficiency in implementation. The quadratic penalty function yields least-square or Euclidean norm approximation 22. The $\ell_{2}$-norm is preferred for energy-efficiency applications. However, the NMPC based on $\ell_{2}$ norm associated to states may also lead to aggressive system behaviour 23]. As an alternative, a systematic way of dealing with large state residuals based on Huber function was proposed in 23. The Huber function, $\phi_{M}(x)$ is equivalent to a $\ell_{2}$-norm within the region $[-M, M]$ and to a $\ell_{1}$-norm outside. The $\ell_{1}$-norm is preferred for robust regulations where the absolute value penalty function so yields $\ell_{1}$-norm approximation. Thus, the sensitivity to outliers or large residuals is lower than the $\ell_{2}$-norm $[22$.

A deadzone-quadratic and deadzone-linear penalty functions that have the 
advantages of $\ell_{2}$ and $\ell_{1}$-norms respectively was proposed in [24]. This method preserved the energy-efficient behaviour within the desired operating zone. The main idea of the deadzone-quadratic penalty function is to assess low penalty or insensitivity for residuals smaller than the deadzone width and quadratic penalty for bigger residuals. This motivates to find a tradeoff between the agile set-point tracking and energy-efficient strategy. This paper is based on the extended Eco-CC system proposed in [21] and 24]. In [21], the capability of the conventional Eco-CC system for the BEVs was enhanced to operate on curvy roads and adapting to the traffic speed limit zones. The $\ell_{2}$-norm penalty function of the formulated NMPC was replaced with the deadzone-quadratic penalty function in 24] and was evaluated with the field experimental tests.

In this paper, a real-time NMPC based on the deadzone-quadratic penalty function with improved constraints handling method is designed for the extended Eco-CC system in the BEVs. For this purpose, the components are considered to develop a system model. First, longitudinal dynamics of the BEV, its energy consumption, as well as road geometry and traffic sign information are modelled in a reasonably accurate framework. Second, a real-time nonlinear receding horizon optimal controller with improved inequality constraints handling and economic penalty function is designed to plan the online cost-effective cruising velocity. Then, the NMPC takes advantage of a convex deadzone-quadratic penalty function for velocity tracking within desired reference zone. The main contribution of this paper is to enhance the NMPC formulation with the states inequality constraints handled with the proposed soft nonlinear complementarity function aimed to preserve the relaxed complementary slackness. The nonlinear complementarity function used in this paper is based on Fischer-Burmeister complementarity function intended to enhance the PMP method. Finally, this paper rigorously evaluates the extended Eco-CC system with numerical simulation and field experimental results. The performance of the system is assessed in terms of energy-efficient velocity regulation and constraints fulfilment.

The remainder of this paper is structured as follows: The system model is introduced in Section 2. The NMPC formulation with deadzone-quadratic 
penalty function and the proposed soft inequity constraint handling method are presented in Section 3 . Section 4 includes the NMPC formulation for the EDAS concept with the numerical simulation and field experimental validation, followed by the conclusion and future work in Section 5 .

\section{Notation}

Throughout this paper, $\mathbb{R}^{n}$ denotes the n-dimensional Euclidean space. $\mathbb{R}_{+}:=$ ${ }_{120}[0, \infty) . \mathbb{N}=\{1,2, \ldots\}$ is set of natural numbers. $\mathbb{N}_{+}:=\mathbb{N} \cup\{0\}$ and $\mathbb{Z}_{[a, b]}:=$ $\{a, a+1, \ldots, b\}$ is set of integers from $a$ to $b$.

\section{System Model}

Safe and energy-efficient velocity profile identification based on the road gradients, and Eco-drive techniques around curves while respecting the traffic signs have a significant improvement in extending the cruising range of the BEVs. The semi-autonomous EDAS concept that extends the functionalities of the Eco-CC system for the BEVs is presented in Figure 1 .

Similar to the modern Eco-CC systems, the driver presets the desired velocity. The semi-autonomous Extended Eco-CC (Ext-Eco-CC) system predictively regulates the velocity with respect to the longitudinal motion of the vehicle, its energy consumption dynamics, road geometric navigation data, and traffic sign information. While the driver handles the steering control of the vehicle, this system should plan a proper energy-efficient cruising velocity profile autonomously for the entire trip without requiring the driver interventions. Even though the vehicle dynamics is modelled in the longitudinal domain, the proposed Ext-Eco-CC system is additionally capable of handling the lateral motion of the vehicle on the curvy roads.

\subsection{Electric Vehicle Dynamics}

The forward motion of the BEV on its moving direction is defined by the sum of all the forces acting on longitudinal direction. The traction force $\left(F_{\text {trac }}\right)$, propels the vehicle forward and acting on the contact area between tires of rear 


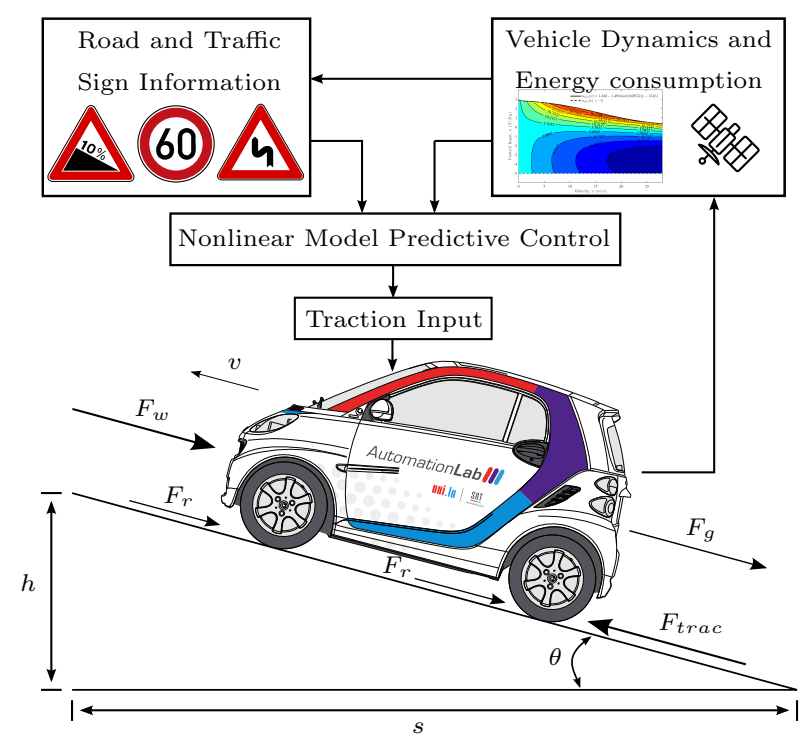

Figure 1: Semi-autonomous Extended Eco-CC System for BEVs

driven wheels and road surface. This force is produced by the powertrain system and transferred power flow from the battery pack to the drive wheels through the electric machine and transmission. On the other hand, there are resistive forces acting against the traction force that attempts to stop the forward motion of the vehicle. The most effective resistive forces generally are the aerodynamic drag $\left(F_{w}\right)$, grading resistance $\left(F_{g}\right)$, and tire rolling resistance $\left(F_{r}\right)$. It is clear that in a downhill motion case, the grading force is in the same direction of traction force and assists the vehicle's propulsion.

The forward one-dimensional motion of vehicle at position, $s$, and velocity, $v$, is assumed as a point mass at the centre of gravity. Hence, its acceleration along the longitudinal direction expressed by Newton's second law of motion as follows:

$$
\begin{aligned}
& \frac{d s}{d t}=v, \\
& \frac{d v}{d t}=\frac{F_{t r a c}-\sum F_{r e s}}{m_{e q}},
\end{aligned}
$$

where $F_{r e s}$ represents the total resistive forces and $m_{e q}$ is the equivalent mass 
of the BEV. The equivalent mass includes kerb weight and rotational inertia of rotating components. Calculation of the equivalent mass requires knowing the values of the mass moments of inertia for all the rotating parts. The equivalent mass of the BEV with unknown values for the inertias of rotating parts can be calculated by an empirical relation as follows:

$$
m_{e q}=m\left(1+\delta_{1}+\delta_{2} i_{g}^{2}\right)
$$

moment of the vehicle, $\delta_{2}$ represents the effect of rotating parts in the powertrain system, and $i_{g}^{2}$ is the single transmission ratio [25].

The power flow from the battery pack passes throughout the inverter which transforms the battery DC voltage to a three-phase AC voltage applicable for the electric machine, single gear ratio gearbox, to the rear wheels or vice versa to enable regenerative braking. The regenerative braking can generate electricity in order to charge the onboard battery pack. The traction force on the reardriven wheels can be expressed as:

$$
F_{\text {trac }}=\frac{i_{g} \eta_{t} \tau_{\text {trac }}}{r_{d}}
$$

where $r_{d}$ is the effective radius of the rear wheel, $\tau_{\text {trac }}$ is the powertrain torque output, and $\eta_{t}$ is the total mechanical efficiency of the transmission between the 155 electric motor output shaft and rear driven wheels. The $\eta_{t}$ is the product of the efficiencies of all the components in the driveline. An average value of the overall mechanical efficiency of the BEVs can be approximated to $90 \%$ 25].

The total resistive forces acting on the vehicle forward motion are the aerodynamic drag $\left(F_{w}\right)$, grading resistance $\left(F_{g}\right)$, and tire rolling resistance $\left(F_{r}\right)$. Hence the $F_{\text {res }}$ can be expressed as,

$$
\sum F_{r e s}=F_{w}+F_{g}+F_{r}
$$

Aerodynamic drag is the fluid drag force between the moving vehicle at velocity $v$ in opposite direction to air in the direction of the fluid free stream flow [25]. 
The aerodynamic drag is a function of vehicle speed $v$ as follows:

$$
F_{w}=\frac{1}{2} \rho_{a} A_{f} C_{D} v^{2}
$$

where $\rho_{a}$ is air density, $A_{f}$ is vehicle frontal area, and $C_{D}$ is the aerodynamic drag coefficient. When a vehicle goes up or down a slope, its weight drive to either opposite or assistant the forward motion. In vehicle performance analysis, the only uphill operation is considered. This grading force is usually called grading resistance [25]. This force can be expressed as:

$$
F_{g}=m_{e q} g \sin (\theta)
$$

where $g=9.81 \mathrm{~m} / \mathrm{s}^{2}$ is the gravitational constant. The rolling resistance is the force resisting the motion when the tire rolls on a surface and mainly caused by hysteresis in the tire materials or the surface (e.g. soil). The rolling resistance has a different and broad range of definitions depending on the application point of view. In this study, the rolling resistance can be expressed as:

$$
F_{r}=\mu_{r r}(v) m_{e q} g \cos (\theta)
$$

where $\mu_{r r}$ is rolling resistance coefficient. In vehicle performance calculation, it is sufficient to consider the rolling resistance coefficient as a linear function of speed as follows:

$$
\mu_{r r}(v)=0.01\left(1+\frac{v}{576}\right) .
$$

This equation predicts the values of $\mu_{r r}(\cdot)$ with acceptable accuracy for speeds up to $v \leq 35.55(\mathrm{~m} / \mathrm{s})[25]$. In this study, the parameters of a commercial BEV is derived from the manufacturer's data sheet and the provided information in [15, 17] for the numerical simulations and field experimental evaluations.

\subsection{Energy Consumption Model}

Energy consumption of a BEV depends on a number of factors including driven velocity, acceleration profile, geometric characteristics of roads, and traffic situations. A detailed exact analytical model for the electric propulsion system of the BEVs including all models and relations of the components with 
unknown parameters can be complex for the EDAS applications. Developing a simple electric vehicle energy model that computes its instantaneous energy consumption is required [26. Hence, a dynamometer test is proposed to be carried out to achieve a relatively simple system identification. Based on test data for a given velocity at a given traction force, the operating point of the electric machine and the related power consumption or regeneration can be determined [21].

The energy consumption during cruising at constant speed is equal to the resistive power. This can be approximated through the curve-fit process with measurement data by a polynomial of velocity as $f_{\text {cruise }}(v)=b_{3} v^{3}+b_{2} v^{2}+$ $b_{1} v+b_{0}$ and acceleration as $f_{a c l}(u)=a_{2} u^{2}+a_{1} u+a_{0}$. Therefore, at any given velocity and control input, a linear relation of the traction power-to-mass ratio can describe the energy consumption of the BEV. The power-to-mass ratio is a performance measurement index of a vehicle, with the power of powertrain output being divided by the mass of the vehicle which is independent of the vehicle's size. Therefore, combining the $f_{\text {cruise }}(v)$ and the $f_{\text {acl }}(u)$, can lead to a model of the power consumption of the BEV. At any given velocity and control input, a linear relation of the traction power-to-mass ratio of the vehicle can be expressed as:

$$
\frac{d e_{c n s}}{d t}=f_{a c l}(u) \frac{p_{t r a c}}{m_{e q}}+f_{c r u i s e}(v) .
$$

where $p_{\text {trac }}=F_{\text {trac }} v$, denotes the traction power. Figure 2 shows power consumption model of a commercial BEV based on traction input and velocity with $98.46 \%$ coefficient of determination (R-squared) achieved through the curve-fit process 21]. Each contour line represents the related power consumption (in $k W)$. At the higher traction input and velocity, the positive energy with the higher rate is consumed. In contrast, at regenerative braking zone at different velocity, a limited amount of energy can be recovered. This model is capable of capturing the energy consumption of a BEV including the regenerative braking for the full-range of velocity and the control input (for more details, see [21]). 


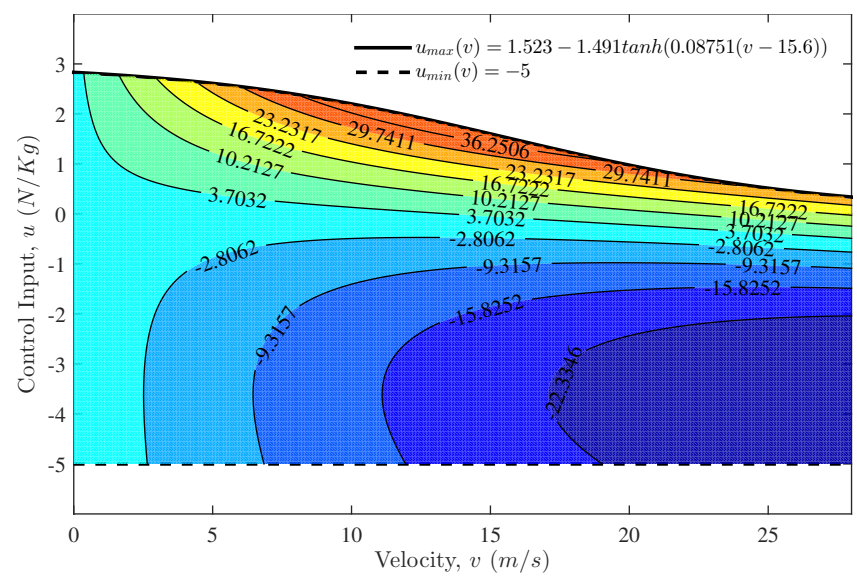

Figure 2: Power consumption of the commercial BEV [21]

\subsection{Road Geometry Profiles and Traffic Sign Models}

The term virtual sensor is used for an information source which is not an actual sensor, but comprises an important input for the EDAS applications [1. One of the most important representatives of this category is the digital road map. The digital map data can be extracted and used by a vehicle when positioning information is available. Standard map positioning techniques are based on the Global Positioning System (GPS) technology. In order to have proper functionality for some of the EDAS applications, the surrounding world such as the road infrastructure should be modelled and interpreted.

One of the most typical ways of representing the road geometric data is to define data points of interest along the road centreline with specified intervals corresponding to the desired road profile accuracy level. There are several interpolation methods to construct new data points of interest and road model estimation. However, most of the methods were introduced in works of literature are applicable to two coordinate system dynamics and are less flexible to be directly applied in a single coordinated EDAS applications. Moreover, the interval determination of data points of interest can lead to either overestimated or underestimated accuracy levels for different road segments. Those approaches impose a tradeoff challenge between accuracy level and fidelity levels 


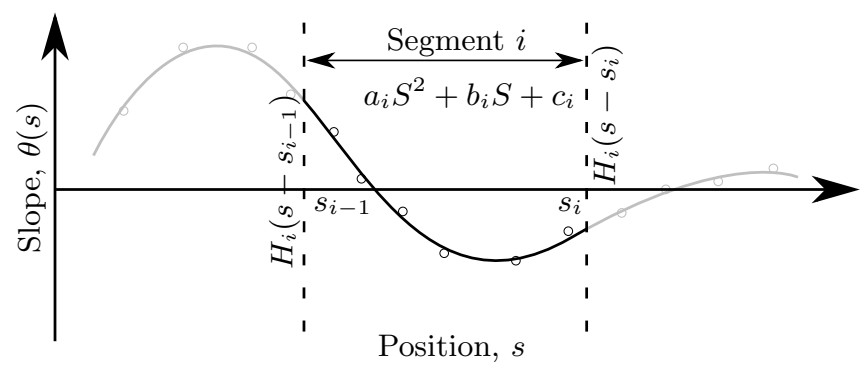

Figure 3: Road slope model at segment $i$ [21]

of the models which may not be desirable for the real-time EDAS applications.

The road slopes, road curvatures, and traffic speed limit zone data are modelled as continuous and differentiable functions in [21]. In that method, the road slope profile $\left(f_{s l p}(\theta(s))\right)$ is proposed to be the sum of quadratic functions of the vehicle position representing each road segments slope data as follows:

$$
f_{s l p}(\theta(s)):=\sum_{n=1}^{N_{s g m}} H_{\left(s-s_{n-1}\right)}^{n}\left(a_{n} s^{2}+b_{n} s+c_{n}\right) H_{\left(s-s_{n}\right)}^{n},
$$

where $N_{s g m}$ is the number of road segments, $H_{\left(s-s_{n-1}\right)}^{n}$ and $H_{\left(s-s_{n}\right)}^{n}$ are hyperfunctions of the $n^{\text {th }}$ road segment. These functions represent the data points in 205 each segment of the road utilising hyper-function concept to interconnect the estimated segments of the road at the boundaries positions, $s_{n-1}$ and $s_{n}$. The modelling concept is shown in Figure 3

The road curves and traffic speed limits profiles are modelled in a similar way [21]. The simple curve is used to express the total absolute road curve profile $\left(f_{\text {crv }}(\delta(s))\right)$ which is defined as:

$$
f_{c r v}(\delta(s)):=\sum_{n=1}^{N_{c r v}} H_{\left(s-s_{e n t}\right)}^{n}\left|\frac{1}{R_{c r v_{n}}(s)}\right| H_{\left(s-s_{e x t}\right)}^{n},
$$

where $N_{c r v}$ is the number of road curves, and $R_{c r v_{n}}$ is the radius of a circle valid for the curve's arc length with two position points, $s_{\text {ent }}$ and $s_{\text {ext }}$, at the respective entrance and exit positions. Furthermore, the traffic speed limit 
profile $\left(f_{l m t}(s)\right)$ can be modelled as:

$$
f_{l m t}(s):=\sum_{n=1}^{N_{l m t}} H_{\left(s-s_{s t r}\right)}^{n}\left(v_{l m t}-v_{\text {max }}\right) H_{\left(s-s_{\text {end }}\right)}^{n}+v_{\text {max }},
$$

where $N_{l m t}$ is the number of speed limit zones, and $v_{l m t}$ is the specified speed limit value at positions starts from $s_{s t r}$ up to the end of the zone $s_{\text {end }}$. The $v_{\max }$ is the maximum speed value of the electric vehicle. This method to model the road geometry and static traffic sign data improve the tradeoff challenge between model complexity and accuracy level (high and low-fidelity models) for the EDAS applications. For more details about the Ext-Eco-CC system see [21, 24].

\section{Nonlinear Model Predictive Control}

A general NMPC formulation is reviewed in this section including the Deadzone penalty function. In addition, the proposed inequality constraint handling technique is introduced which aims to enhance the PMP method.

\subsection{Optimal Control Problem}

Consider a general discrete-time system:

$$
x_{t+1}=f\left(x_{t}, u_{t}\right),
$$

where $t \in \mathbb{N}_{+} ; x_{t} \in \mathbb{R}^{n_{x}}$ is the system states vector, and $u_{t} \in \mathbb{U} \subset \mathbb{R}^{n_{u}}$ is a nonempty measurable set for the inputs. The $f(\cdot)$ is nonlinear Borel-measurable vector of functions that describes the system dynamics. Let $N \in \mathbb{N}$ be the both state and control prediction horizon. Define an $\mathrm{N}$-stage feedback control policy as:

$$
\boldsymbol{\pi}:=\left\{\pi_{0}(\cdot), \pi_{1}(\cdot), \ldots, \pi_{N-1}(\cdot)\right\},
$$

where the Borel-measurable function $\pi_{i}(\cdot): \mathbb{R}^{(i+1) n_{x}} \rightarrow \mathbb{U}$, for all $i=0, \ldots, N-1$ is a general state feedback control law. The control input $u_{i}$ is selected as the feedback control law $u_{i}=\pi_{i}(\cdot)$ at the $i^{\text {th }}$ stage of the control policy. In receding 
horizon optimal control, the cost function of an Optimal Control Problem (OCP) is commonly defined as:

$$
V_{N}\left(x_{t}, \boldsymbol{\pi}\right):=\sum_{i=0}^{N-1} J_{c}\left(\hat{x}_{i}, u_{i}\right)+J_{f}\left(\hat{x}_{N}\right),
$$

where $J_{c}: \mathbb{R}^{n_{x}} \times \mathbb{U} \rightarrow \mathbb{R}_{+}$and $J_{f}: \mathbb{R}^{n_{x}} \rightarrow \mathbb{R}_{+}$are the cost-per-stage function and the final cost function, respectively. The $\hat{x}_{i}$ denotes the predicted states at time $i$ given the initial states $\hat{x}_{0}=x_{t}$, and control law $\left\{\pi_{i}(\cdot)\right\}_{i=0}^{N-1}$.

Using the cost function (16), the OCP for (14) is formulated as follows:

$$
V_{N}^{*}\left(x_{t}\right):=\underset{\pi}{\operatorname{minimise}} \quad V_{N}\left(x_{t}, \boldsymbol{\pi}\right)
$$

subject to:

$$
\begin{array}{ll}
\hat{x}_{i+1}=f\left(\hat{x}_{i}, \pi_{i}\right), & \text { for all } i \in \mathbb{Z}_{[0, N-1]}, \\
\pi_{i}(\cdot) \in U, & \text { for all } i \in \mathbb{Z}_{[0, N-1]}, \\
g_{j}\left(\hat{x}_{i}, \pi_{i}\right) \leq 0, & \text { for all } j=1, \ldots, s, \text { and } i \in \mathbb{Z}_{[0, N-1]}, \\
\hat{x}_{0}=x_{t}, &
\end{array}
$$

where $V_{N}^{*}\left(x_{t}\right)$ denotes the optimal value function under the optimal control policy $\pi^{*}$. The inequality state constraints are denoted by $g_{j}\left(\hat{x}_{i}, \pi_{i}\right)$ which 225 are required to be satisfied. The OCP in receding horizon principle involves applying the first element of the control action sequence $u_{t}=\pi_{0}^{*}(\cdot)$ repeatedly to the system at each sampling time.

The first-order necessary conditions for a solution in the OCP 17 to be optimal are based on Karush-Kuhn-Tucker (KKT) conditions as follows:

$$
\begin{aligned}
\nabla V_{N}\left(x^{*}, \pi^{*}\right)+\nabla \lambda^{*} f\left(x^{*}, \pi^{*}\right) & +\nabla \mu^{*} g\left(x^{*}, \pi^{*}\right)=0, \\
f\left(x^{*}, u^{*}\right) & =0, \\
g_{j}\left(x^{*}, u^{*}\right) & \leq 0, \\
\mu_{j}^{*} & \geq 0, \\
\mu_{j}^{*} g_{j}\left(x^{*}, u^{*}\right) & =0, \text { for all } j \in \mathbb{Z}_{\left[1, n_{g}\right]},
\end{aligned}
$$

where the gradient symbol $\nabla$ is the transpose of the Jacobian i.e. $\nabla g(x):=$ $\frac{\partial g(x)^{T}}{\partial x}$. Condition $18 \mathrm{e}$ is also called complementary slackness condition. It 
can be interpreted as if the $j^{\text {th }}$ inequality constraint of the primal problem is inactive at the optimum $g_{j}\left(x^{*}, \pi^{*}\right) \leq 0$, then the $j^{\text {th }}$ dual variable has to be zero $\left(\mu_{j}^{*}=0\right)$. The KKT approach to the OCP generalises the method of Lagrange multipliers, which allows only equality constraints (for more details see e.g., [27]).

\subsection{Real-time Nonlinear Model Predictive Control}

One of the general approaches to the NMPC is to transform the underlying optimisation problem into equality constrained root finding problem that is solved based on Newton-type method through necessary conditions for optimality 28. The inequality constraints are generally handled based on Interior Point (IP) reformulation of the objective function. Indirect methods based on the PMP are efficient to solve the OCP with high accuracy demands which this leads to Two-Point Boundary-Value Problem (TP-BVP). The achieved nonlinear TP-BVP can be solved numerically with the real-time algorithms.

Considering the indirect method based NMPC solvers, Continuation / Generalized Minimal RESidual (C/GMRES) is a fast numerical algorithm for nonlinear receding horizon control which was proposed by [29]. Similar to the Newton-type controller, the C/GMRES method performs only one Newton-type iteration in each sampling time and is based on a sequential formulation.

Let's consider a continuous-time system and assume that every function is differentiable as many times as necessary. The state equation and an $n_{c^{-}}$ dimensional equality constraint are given as:

$$
\dot{x}=f(x(t), u(t)), \quad C(x(t), u(t))=0 .
$$

An inequality constraint can be converted into equality constraint by introducing a dummy input based on auxiliary variable method [29]. The following performance index with the initial state given by the actual state $x(t)$ is minimised:

$$
V_{T}(x(t), u(t))=\int_{t}^{t+T} J_{c}\left(x\left(t^{\prime}\right), u\left(t^{\prime}\right)\right) d t^{\prime}+J_{f}(x(t+T)) .
$$


The optimal control $u^{*}\left(t^{\prime} ; t, x(t)\right)$ that minimises $V_{T}(x(t), u(t))$ is computed over prediction horizon $t^{\prime} \in[t, t+T]$. The NMPC problem is essentially a family of finite-horizon optimal control problems along a fictitious time $\tau$ as follows:

$$
V_{T}^{*}(x(t)):=\underset{u^{*}}{\operatorname{minimise}} \int_{0}^{T} J_{c}\left(x^{*}(\tau, t), u^{*}(\tau, t)\right) d \tau+J_{f}\left(x^{*}(T, t)\right)
$$

subject to:

$$
\begin{aligned}
& x_{\tau}^{*}(\tau, t)=f\left(x^{*}(\tau, t), u^{*}(\tau, t)\right), \\
& C\left(x^{*}(\tau, t), u^{*}(\tau, t)\right)=0, \\
& x(0, t)=x(t),
\end{aligned}
$$

where subscript $\tau$ denotes partial differentiation with respect to $\tau$ [30]. The new state vector $x^{*}(\tau, t)$ represents a trajectory along the $\tau$ axis starting from $x(t)$ at $\tau=0$ and the prediction horizon $T$ is a function of time, $T=T(t)$ in general 31.

Let $H$ denotes the Hamiltonian defined by:

$$
H(x, \lambda, u, \mu)=J_{c}(x, u)+\lambda^{\mathrm{T}} f(x, u)+\mu^{\mathrm{T}} C(x, u),
$$

where $\lambda \in \mathbb{R}^{n_{x}}$ denotes the costate, and $\mu \in \mathbb{R}^{n_{c}}$ denotes the Lagrange multipliers associated with equality constraints. The first-order conditions necessary for the OCP are obtained by the calculus of variation as the Euler-Lagrange equations [31]:

$$
\begin{array}{ll}
x_{\tau}^{*}=f\left(x^{*}, u^{*}\right), & x^{*}(0, t)=x(t), \\
\lambda_{\tau}^{*}=-H_{x}^{\mathrm{T}}\left(x^{*}, \lambda^{*}, u^{*}, \mu^{*}\right), & \lambda^{*}(T, t)=J_{f}^{\mathrm{T}}\left(x^{*}(T)\right), \\
H_{u}^{\mathrm{T}}\left(x^{*}, \lambda^{*}, u^{*}, \mu^{*}\right)=0, & \\
C\left(x^{*}, u^{*}\right)=0 . &
\end{array}
$$

The control input $u^{*}$ and the Lagrange multiplier $\mu^{*}$ at each time $\tau$ on the prediction horizon are determined from $x^{*}$ and $\lambda^{*}$ by algebraic equations $H_{u}(\cdot)=0$ and $C(\cdot)=0$. The nonlinear TP-BVP has to be solved within the sampling period for the measured state $x(t)$ at each sampling time, which is one of the major difficulties in NMPC [31]. 
The control input function over the prediction horizon is regarded as the unknown quantity in the TP-BVP. In order to represent the unknown control input function with a finite number of parameters, we discretise the prediction horizon of the optimal control problem into $N$ steps. Then, the discretised conditions for optimality are given as follows:

$$
\begin{array}{ll}
x_{i+1}^{*}(t)=x_{i}^{*}(t)+f\left(x_{i}^{*}(t), u_{i}^{*}(t)\right) \Delta \tau, & x_{0}^{*}(t)=x(t), \\
\lambda_{i}^{*}=\lambda_{i+1}^{*}-H_{x}^{\mathrm{T}}\left(x_{i}^{*}(t), \lambda_{i+1}^{*}(t), u_{i}^{*}(t), \mu_{i}^{*}(t)\right) \Delta \tau, & \lambda_{N}^{*}(t)=J_{f}^{\mathrm{T}}\left(x_{N}^{*}(t)\right), \\
H_{u}^{\mathrm{T}}\left(x_{i}^{*}(t), \lambda_{i+1}^{*}(t), u_{i}^{*}(t), \mu_{i}^{*}(t)\right)=0, & \\
C\left(x_{i}^{*}(t), u_{i}^{*}(t)\right)=0 &
\end{array}
$$

where $\Delta \tau:=T / N$. On the discretised horizon, sequences of the state, costate, input, and Lagrange multiplier associated with the equality constraint are denoted by $\left\{x_{i}^{*}(t)\right\}_{i=0}^{N},\left\{\lambda_{i}^{*}(t)\right\}_{i=0}^{N},\left\{u_{i}^{*}(t)\right\}_{i=0}^{N-1},\left\{\mu_{i}^{*}(t)\right\}_{i=0}^{N}$, respectively. As a result, NMPC is formulated as a discrete-time TP-BVP (24) for a measured state $x(t)$ at time $t[31$.

Let us define vector $U(t) \in \mathbb{R}^{n N}\left(n:=n_{u}+n_{c}\right)$ composed of the sequences of the input vectors and multipliers as follows:

$$
U(t):=\left[u_{0}^{* \mathrm{~T}}(t), \mu_{0}^{* \mathrm{~T}}(t) \cdots u_{N-1}^{* \mathrm{~T}}(t), \mu_{N-1}^{* \mathrm{~T}}(t)\right]^{\mathrm{T}} .
$$

The sequences of $\left\{x_{i}^{*}(t)\right\}_{i=0}^{N}$ and $\left\{\lambda_{i}^{*}(t)\right\}_{i=0}^{N}$ can be regarded as functions of $U(t)$ and $x(t)$. Then, the optimality conditions 24 can be regarded as an $n N$-dimensional equation system given by:

$$
F(U(t), x(t), t):=\left[\begin{array}{c}
H_{u}^{\mathrm{T}}\left(x_{0}^{*}, \lambda_{1}^{*}, u_{0}^{*}, \mu_{0}^{*}\right) \\
C\left(x_{0}^{*}, u_{0}^{*}\right) \\
\vdots \\
H_{u}^{\mathrm{T}}\left(x_{N-1}^{*}, \lambda_{N}^{*}, u_{N-1}^{*}, \mu_{N-1}^{*}\right) \\
C\left(x_{N-1}^{*}, u_{N-1}^{*}\right)
\end{array}\right]=0,
$$

where $F$ depends on $t$ when the horizon length $T$ is time dependent [31]. Solving (26) at each time by the iterative methods such as Newton's method is computationally expensive and thus inefficient. Instead, the continuation method is 
applied considering the real time $t$ as the continuation parameter. That is, the time derivative of $U$ obtained so that 26 is satisfied identically. If the initial solution $U(0)$ of the problem is determined so as to satisfy $F(U(0), x(0), 0)=0$, then we can trace $U(t)$ by integrating $\dot{U}(t)$ fulfilling the condition:

$$
\dot{F}(U(t), x(t), t)=A_{s} F(U(t), x(t), t),
$$

where $A_{s}$ is a positive real number. The right-hand side of 27 stabilizes $F=0$. Equation 27 is equivalent to a linear equation with respect to $\dot{U}(t)$ given by:

$$
\dot{U}=F_{U}^{-1}\left(-A_{s} F-F_{x} \dot{x}-F_{t}\right) .
$$

If the matrix $F_{U}$ is nonsingular, 28 is solved efficiently by the GMRES [32, one of the Krylov subspace methods for linear equations. We can update the

There are several works of literature that systematically transform a general inequality constrained into the surrogate equality constraints by various methods. For instance, barrier methods are one of the main IP methods that convert the inequality constraints 17 into equality constraints by utilising a barrier 
function $\Phi_{g}(\cdot): \mathbb{R}^{n_{g}} \rightarrow \mathbb{R}$ as follows:

$$
V_{N}^{*}\left(x_{t}, \gamma\right):=\underset{\pi}{\operatorname{minimise}} \quad V\left(x_{t}, \boldsymbol{\pi}\right)+\gamma \Phi_{g}\left(x^{*}(\tau, t), u^{*}(\tau, t)\right)
$$

subject to:

$$
\begin{aligned}
& x_{\tau}^{*}(\tau, t)=f\left(x^{*}(\tau, t), u^{*}(\tau, t)\right), \\
& C\left(x^{*}(\tau, t), u^{*}(\tau, t)\right)=0, \\
& x(0, t)=x(t),
\end{aligned}
$$

where $\gamma$ is barrier parameter. The $\Phi_{g}(\cdot)$ must take on the value $+\infty$ whenever $g_{j}(\cdot)>0$ for some $j$ and a finite value otherwise. The indicator function:

$$
I_{g}(\hat{x}, u):= \begin{cases}0 & \text { if } g_{j}(\hat{x}, u) \leq 0, \\ +\infty & \text { otherwise }\end{cases}
$$

trivially achieves the purpose of a barrier. However, the barrier function is required to be convex and continuously differentiable [27. One of the most well-known continuous and twice differentiable function that approximates 30 is logarithmic barrier function as follows:

$$
\Phi_{g}(\hat{x}, u):=-\sum_{j=1}^{n_{g}} \ln \left(-g_{j}(\hat{x}, u)\right),
$$

with domain $\left\{\hat{x} \in \mathbb{R}^{n_{x}} \mid g_{j}(\hat{x}, u) \leq 0, \forall j=1, \ldots, n_{g}\right\}$.

A modified type of barrier method is Exterior Penalty method. In this method, an exterior penalty function is included in the cost function on the constraint violations. The Exterior penalty function is defined as:

$$
\Phi_{e}(\hat{x}, u):=\left\{\begin{array}{lc}
0 & \text { if } \quad h_{j}(\hat{x}, u) \leq 0, \\
w_{j} h_{j}(\hat{x}, u)^{2} & h_{j}(\hat{x}, u) \geq 0,
\end{array}\right.
$$

where $w_{j}>0$ is the penalty weight of the $j^{\text {th }}$ constraint [28].

Auxiliary Variable method is an alternative type of inequality constraints handling introduced with an additional optimisation variable to transform the inequality constraints into equality constraints. Let $\eta \in \mathbb{R}^{n_{g}}$ be a vector of the 
auxiliary variables, the inequality constraint $g_{j}(\cdot)$ can be transformed into the equality constraint as follows:

$$
g_{j}(\hat{x}, u)+\eta_{j}^{2}=0 .
$$

A penalty term $-w_{j} \eta_{j}$ is included in the cost function to avoid singularity [29].

If the solution of the OCP 29 is very close to the constraint boundary, where the barrier function is increasing extremely quickly, and as a result, the optimisation problem becomes very ill-conditioned. One of the most effective IP methods capable of dealing with ill-conditioning is so-called primal-dual methods. These algorithms operate in both the primal and dual space and find solutions to the primal OCPs and their duals simultaneously. Primal-dual methods are more efficient in practice than primal barrier methods since they generate different search directions using also information from the dual space, and therefore iterates generated by the two algorithms do not coincide in general [27.

Let's consider the OCP (17) and its first-order necessary conditions (18), in order to account complementarity conditions in the necessary conditions and avoid the ill-conditioning, a semi-smooth transformation can be utilised. One of the most widely used examples of complementarity functions is the semi-smooth Fischer-Burmeister $(\mathrm{FB})$ function introduced in [35]. The FB function $\left(\Phi_{F B}(\cdot)\right)$ which is used in this study given as follows:

$$
\Phi_{F B}\left(\mu_{j}, g_{j}(\hat{x}, u)\right)=\sqrt{\mu_{j}^{2}+g_{j}(\hat{x}, u)^{2}}-\left(\mu_{j}-g_{j}(\hat{x}, u)\right) .
$$

Complementarity functions provide a convenient tool for converting problems that involve complementarity conditions into equations [36].

The FB function is differentiable everywhere except at the $\left(\mu_{j}, g_{j}(\hat{x}, u)\right)=$ $(0,0)$. Several works of literature were proposed to construct smoothing functions of FB function. For instance, the Kanzow smooth FB function was proposed in [37] as follows:

$$
\Phi_{S F B}\left(\mu_{j}, g_{j}(\hat{x}, u)\right)=\sqrt{\mu_{j}^{2}+g_{j}(\hat{x}, u)^{2}+2 \epsilon}-\left(\mu_{j}-g_{j}(\hat{x}, u)\right),
$$


where the 35 was replaced by $\epsilon$ or $\epsilon^{2}$ proposed by [38 and [39. In addition, a smoothing inexact Newton method was presented for solving nonlinear complementarity problems by [40. The smoothed FB function is continuously differentiable and if $\epsilon \rightarrow 0$, then the smooth FB function (35) coincides with the FB function (34).

The optimiser may face an infeasible problem that can lead to a serious problem with the predictive control problem due to the hard implementation of inequality constraints. This might happen due to an unexpectedly large disturbance or the real plant behaves differently from the internal model. An effective strategy is required to handle the infeasibility. One systematic strategy for dealing with infeasibility is to "soften" the constraints [4]. The FB function is a hard constraint implementation of the $g_{j}(\hat{x}, u) \leq 0$ which leads to infeasibility in case of constraint violation. In this paper, a soft constraint implementation based on the FB function is proposed to address the shortcoming of the smoothed FB function as follows:

$$
\hat{\Phi}_{S F B}\left(\mu_{j}, g_{j}(\hat{x}, u)\right)=\sqrt{(1-\epsilon) \mu_{j}^{2}+g_{j}(\hat{x}, u)^{2}+2 \epsilon}-\left((1+\epsilon) \mu_{j}-g_{j}(\hat{x}, u)\right) .
$$

The introduction of the $\epsilon$ as a smoothing parameter transform the nonsmooth problems into a smooth problem. The orthogonality of the vectors $\mu_{j}$ and $g_{j}(\hat{x}, u)$ is relaxed by $(1 \pm \epsilon)$ to prevents the Lagrange multipliers approach to infinity in dual space due to constraint violation. Therefore, this opens the way to use the hard and soft inequality constraints in the OCP with the complementary conditions to address the shortcoming of the PMP method.

\subsection{Deadzone Penalty Functions}

In many practical NMPC applications considering the energy-efficiency, it is desirable to reach a region of reference set-points with relatively low-cost value rather than costly but accurate and agile set-point tracking. This could be accomplished using a nonnegative and symmetric deadzone-quadratic penalty 
function such as:

$$
\phi_{q}(x):= \begin{cases}0 & :|x| \leq z, \\ x^{2}-z^{2} & :|x|>z,\end{cases}
$$

where $z$ is the edge of free zone that no penalty is assessed if $|x| \leq z$. The $\phi_{q}(\cdot)$ function agrees with least-square for any residual outside of the zone width. In other words, the residuals smaller than the zone width are ignored which lead to low-cost function value.

In a case of energy-efficient robust regulations, deadzone-linear penalty function agrees with absolute value for the residual outside of the zone width as follows:

$$
\phi_{l}(x):= \begin{cases}0 & :|x| \leq z, \\ |x|-z & :|x|>z .\end{cases}
$$

Unfortunately, these deadzone penalty functions are not differentiable and continuous which lead to a challenging OCPs. However, a smooth approximation of deadzone penalty function may address the challenge.

In this paper, a deadzone penalty function based on softplus rectifier is adapted 24]. The softplus is an approximation to the activation function socalled Rectified Linear Unit (ReLU) which is mostly utilised in the deep neural networks [42]. The proposed deadzone-linear penalty function is a combination of the two softplus as follows:

$$
\psi_{l}(x):=\ln (1+\exp (x-z))+\ln (1+\exp (-x-z))
$$

The $\psi_{l}(x)$ have advantages such as being a convex function with efficient computation and gradient propagation [43]. The gradient of the deadzone-linear penalty function is a combination of two sigmoid functions as follows:

$$
\frac{d \psi_{l}(x)}{d x}=\frac{\exp (x-z)}{1+\exp (x-z)}-\frac{\exp (-x-z)}{1+\exp (-x-z)} .
$$

Similar to $\psi_{l}(x)$, the deadzone-quadratic penalty function can be formulated as follows:

$$
\psi_{q}(x):=(\ln (1+\exp (x-z))+\ln (1+\exp (-x-z)))^{2}
$$




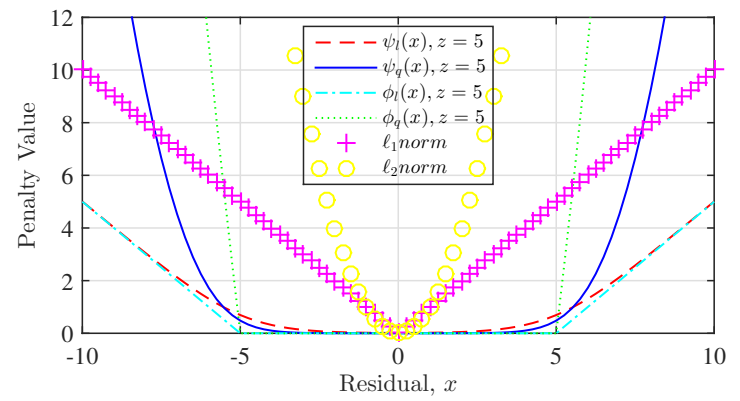

Figure 4: Deadzone Penalty Functions with Relative Comparisons 24

The gradient of the deadzone-linear penalty function is a linear continuous function with a deadzone area, $[-z, z]$, as follows:

$$
\frac{d \psi_{q}(x)}{d x}=2 \psi_{l}(x) \frac{d \psi_{l}(x)}{d x} .
$$

For sake of simplicity, Figure 4 shows the proposed $\psi_{q}(x)$ and $\psi_{l}(x)$ penalty functions for a scalar residual with $z=5$ in comparison with $\phi_{q}(x), \phi_{l}(x), \ell_{2}$, and $\ell_{1}$-norms. Note that when the state residual is within the zone, the gradient is non-zero and the optimality conditions are satisfied as $\ell_{1}$ and $\ell_{2}$-norms. In other words, the states will converge to final reference set-point values but slower than conventional norms which leads to the energy-efficient behaviour.

\section{Case Study: System Design and Evaluation}

The proposed NMPC for the Ext-Eco-CC system has been designed and evaluated with numerical simulation as well as practical experiments on a test track using realistic values of the parameters. A Smart Electric Drive third generation (Smart-ED) commercial BEV, which is available for practical experiments, is chosen here to model the dynamics of a BEV and its energy consumption (Figure5). A closed test track located at Colmar-Berg, Luxembourg, (CFC) is chosen to model the road geometry with traffic information (Figure 6). The test track has a total length of $1.255 \mathrm{~km}$ and includes curves and relative slope profile. This track has four main curves with $20 \mathrm{~m}, 25 \mathrm{~m}, 15 \mathrm{~m}$, and $27 \mathrm{~m}$ radius, respectively. The straight road segments are considered to have a nearly infinite 


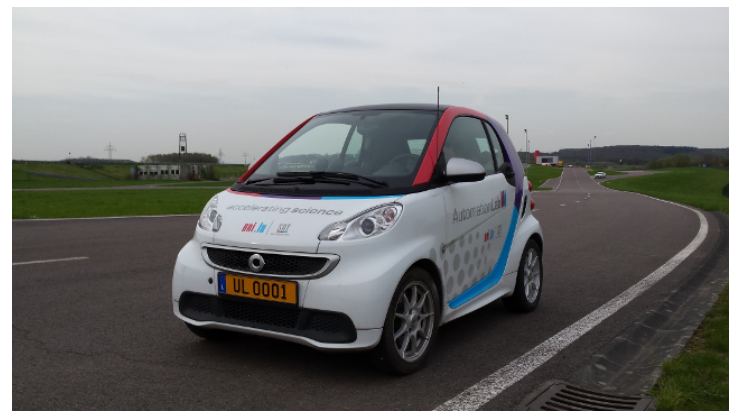

Figure 5: Smart Fortwo Electric Drive 24]

radius. In addition, a speed limit $v_{l m t}=22.23 \mathrm{~m} / \mathrm{s}$ zone is assumed between positions $500 \leq s \leq 850$. The test track slope profile, $f_{s l p}(\theta(s))$, is fitted within nine segments with $98.93 \%$ coefficient of determination [21].

\subsection{Case Study: NMPC for Ext-Eco-CC}

The state vector for the Ext-Eco-CC system from Eqs. (1), 22), and (10), is defined as $x_{t}=\left[s, v, e_{c n s}\right]^{T} \in \mathbb{R}^{3}$; the control input is the traction input applied on BEV, $u_{t}=u \in \mathbb{U} \subset \mathbb{R}$ (for more details see [21]); Please note that all states are measurable and the measurement noise is negligible.

The cost-per-stage function for the Ext-Eco-CC system is defined as:

$$
J_{c}\left(\hat{x}_{i}, u_{i}\right):=\sum_{i=0}^{N-1} \frac{1}{2}\left[\psi_{q}\left(\hat{x}_{i}-x_{r e f}\right)_{Q}+\left\|u_{i}-u_{r e f}\right\|_{R}^{2}\right]+w_{e} \hat{e}_{c n s_{i}},
$$

with corresponding weights $\left(Q, R, w_{e}\right)$. The final cost function for Ext-Eco-CC

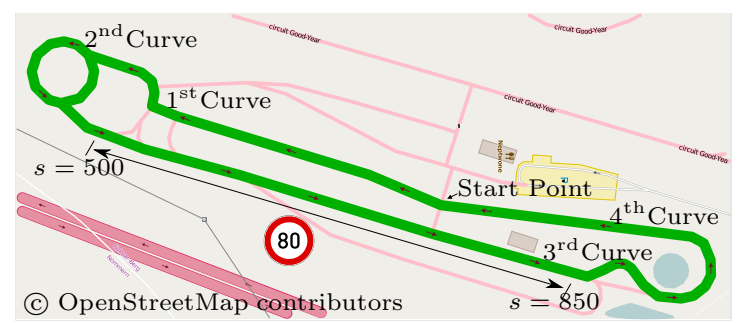

Figure 6: Centre de Formation pour Conducteurs (CFC) 44 
system is defined as:

$$
J_{f}\left(\hat{x}_{N}\right):=\frac{1}{2} \psi_{q}\left(\hat{x}_{N}-x_{r e f}\right)_{Q}
$$

The control input is limited by:

$$
u_{\min }(v) \leq u \leq u_{\max }(v)
$$

where $u_{\min }(v)$ and $u_{\max }(v)$ can be identified based on the traction-velocity map of the BEV. The limit $u_{\max }(v)$ is identified as:

$$
u_{\max }(v)=c_{1}-c_{2} \tanh \left(c_{3}\left(v-c_{4}\right)\right),
$$

where the constants are identified as $c_{1}=1.523, c_{2}=1.491, c_{3}=0.08751$, and $c_{4}=15.6$ with $99.74 \%$ coefficient of determination. The maximum hybrid brake system control input is chosen to be constant, $u_{\min }(v)=-5+c_{5} v(N / K g)$ $\left(c_{5}=0\right)$, which is limited to a stable slip ratio region to avoid the wheels from locking up [21].

The lateral acceleration of the BEV should be lower than the comfort level $\left(\hat{\Psi}_{r e f}\right)$ by an inequality constraint as follows:

$$
g_{1}\left(\hat{s}_{i}, \hat{v}_{i}\right):=\hat{v}_{i}^{2} / f_{\text {crv }}\left(\delta\left(\hat{s}_{i}\right)\right) \leq \hat{\Psi}_{r e f},
$$

where the expected lateral acceleration is based on longitudinal velocity and road curvature map. The velocity of the BEV should also be lower than speed limit zones as:

$$
g_{2}\left(\hat{s}_{i}, \hat{v}_{i}\right):=\hat{v}_{i} \leq f_{l m t}\left(\hat{s}_{i}\right) .
$$

In addition, the velocity should be within the standstill and the reference setpoint so-called funnel concept (see e.g., [4]) as follows:

$$
g_{3}\left(\hat{v}_{i}\right):=0 \leq \hat{v}_{i} \leq v_{r e f}+v_{r l x}
$$

where $v_{r e f}$ is the reference set-point and $v_{r l x}$ is the relaxed velocity for the inequality constraint. The energy consumption of the BEV should be less than the permitted maximum amount as follows:

$$
g_{4}\left(\hat{e}_{c n s_{i}}\right):=\hat{e}_{c n s_{i}} \leq e_{c n s_{r e f}}+e_{c n s_{r l x}}
$$


where $e_{c n s_{r e f}}$ and $e_{c n s_{r l x}}$ are the reference energy consumption and its relaxed value, respectively. The inequality constraints of the Ext-Eco-CC system is implemented based on the proposed soft FB function. The numerical method to solve the obtained NMPC formulation for the Ext-Eco-CC system is based on the PMP utilising the real-time C/GMRES algorithm.

\subsection{Numerical Simulations}

Performance of the proposed NMPC for the Ext-Eco-CC system is evaluated by numerical simulation. The reaction of the predictive controller for the road slope, the road curve, and the speed limit zone is investigated separately. The prediction horizon of the NMPC is chosen as $T=10 \mathrm{~s}$ to cover the upcoming road variations. This prediction horizon is discretised into $N=20$ steps. The reference velocity is chosen as $\left(v_{r e f}=20 \mathrm{~m} / \mathrm{s}\right)$.

Figure 7 shows the performance of the NMPC on the straight hilly road with positive slope. The road has a slope profile shown in Figure 7a indicating an up hill step in road elevation. Figure $7 \mathrm{~b}$ shows the velocity profiles of the NMPC with and without considering energy consumption (NMPC vs Eco-NMPC). The $\mathrm{BEV}$ starts from the standstill to reach the reference velocity. The BEV speeds up before the uphill to use the kinetic of the vehicle to overcome the up-slope. The BEV slows down during the climbing the hill in an energy efficient manner. Afterwards, the BEV track the velocity reference on a straight flat road. The influence of the predictive controllers on the energy consumption of the BEV is shown in Figure $7 \mathrm{c}$. In total, the Eco-NMPC is approximately $+10 \%$ more energy efficient in comparison to the NMPC.

The performance of the NMPC in both ecological modes dealing with a $25 \mathrm{~m}$ radius curvy road is shown in Figure 8 . Figure 8 a shows the BEV speeds up from the standstill to reach the reference velocity. The BEV slows down in advance to enter the curve $\left(s_{h}=900 \mathrm{~m}\right)$ below the maximum allowed lateral acceleration. The BEV track the safe velocity during the curve and speeds up after the curve at the exit position $\left(s_{h}=1100 \mathrm{~m}\right)$. It is shown that the Eco-NMPC has the 
(a)

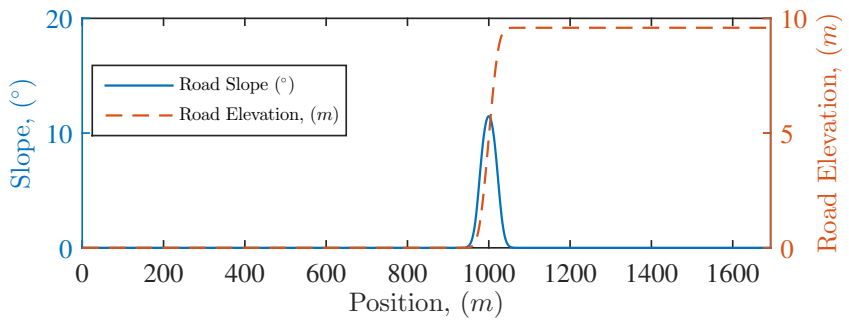

(b)
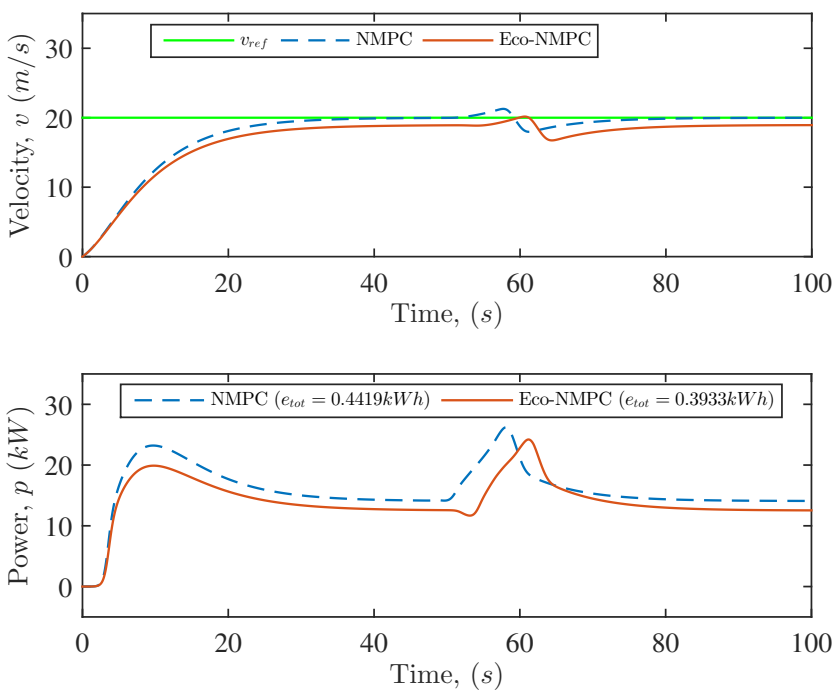

Figure 7: Performance of the NMPC on the straight hilly road with positive slope; (a) road slope profile, (b) velocity profile, (c) energy consumption profile. 
(a)
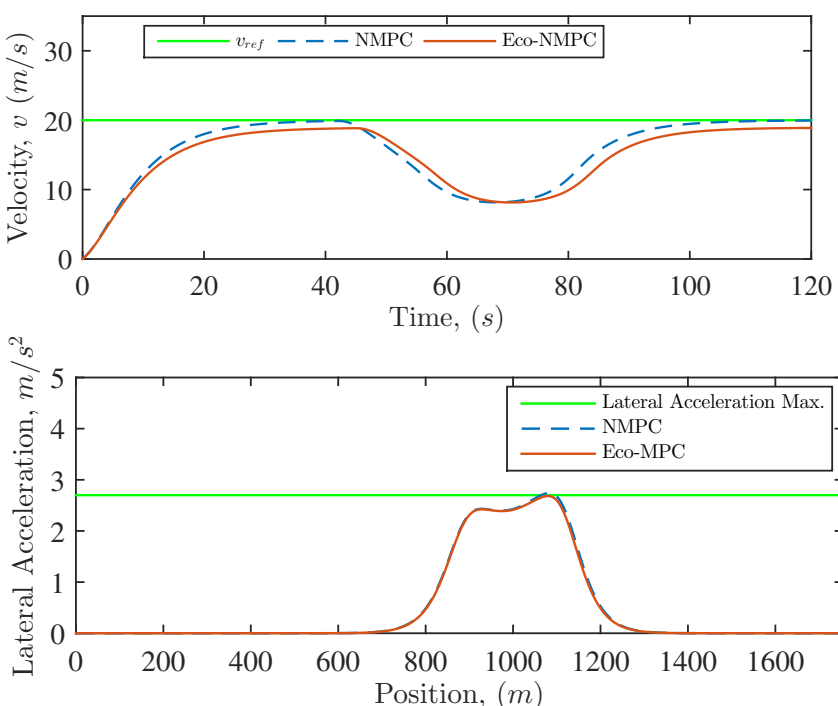

(c)

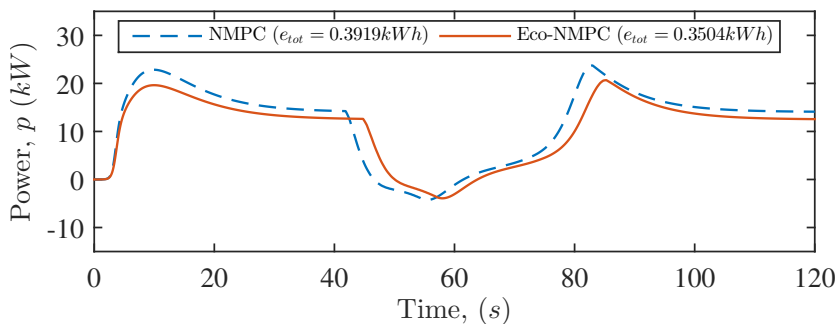

Figure 8: Performance of the NMPC on the curvy road; (a) velocity profile, (b) lateral acceleration profile, (c) energy consumption profile.

technique during the curve. The lateral acceleration profile for the both NMPC and Eco-NMPC are shown in Figure $8 \mathrm{c}$ with a similar reaction to the curvy road. In total, the Eco-NMPC is approximately $+10.5 \%$ more energy efficient in comparison to the NMPC. It is shown that energy efficient velocity profile, in general, is not necessarily could be achieved by slower velocity profile.

Figure 9 shows the performance of the NMPC on the straight road with a speed limit zone $\left(v_{l m t} \leq 10 \mathrm{~m} / \mathrm{s}\right)$. The velocity profile generated by the NMPC and Eco-NMPC are converging to the reference velocity $\left(v_{r e f}=20 \mathrm{~m} / \mathrm{s}\right)$ outside the speed limit zone as shown in Figure 9a. The both NMPC satisfy the speed limit zone constraint. Figure $9 \mathrm{~b}$ shows the power consumption of the both 


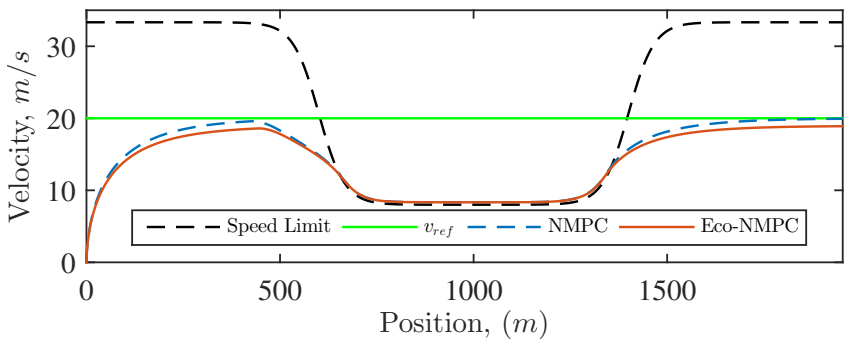

(a)

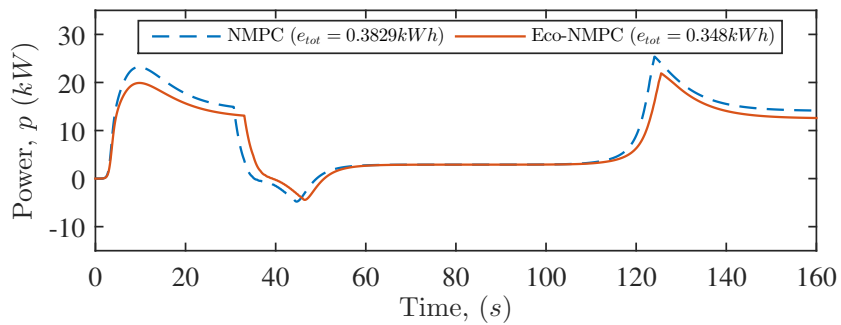

Figure 9: Performance of the NMPC on the straight road with speed limit zone; (a) velocity profile, (b) energy consumption profile.

NMPCs. In total, the Eco-NMPC is approximately $+9 \%$ more energy efficient in comparison to the NMPC in this simulation scenario. Similar to the ecological drive around the curve, the Eco-NMPC shows almost identical behaviour in comparison to the NMPC within the speed limit zone.

385

\subsection{Field Experiments}

In order to validate the proposed EDAS concept, the NMPC with deadzonequadratic penalty function is experimentally implemented on the Smart-ED vehicle and the Ext-Eco-CC system is tested on the CFC test track. The position of the Smart-ED is updated by the GPS sensor. The velocity and energy conis updated by the Controller Area Network (CAN-bus) through the On-Board Diagnose (OBD) interface. The onboard computational resource for the ExtEco-CC concept is foreseen by a Linux operating system on the Intel ${ }^{\circledR}$ Core $^{\text {TM }}$ i7 with a memory of $7.7 \mathrm{GiB}$ PC and a connection panel. The connection panel 395 10 . 


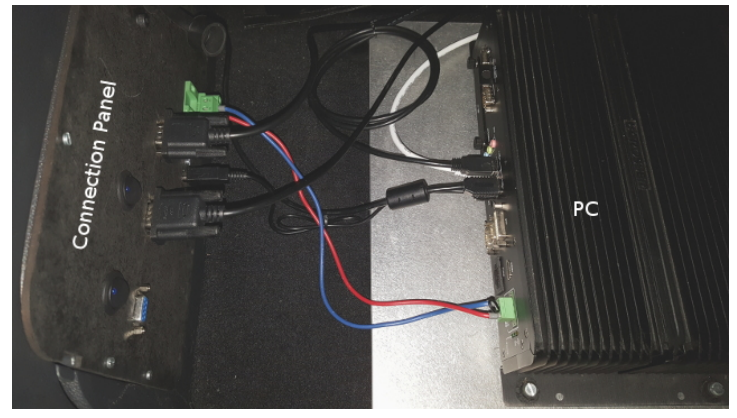

Figure 10: The Linux operated PC with Connection Panel [24]

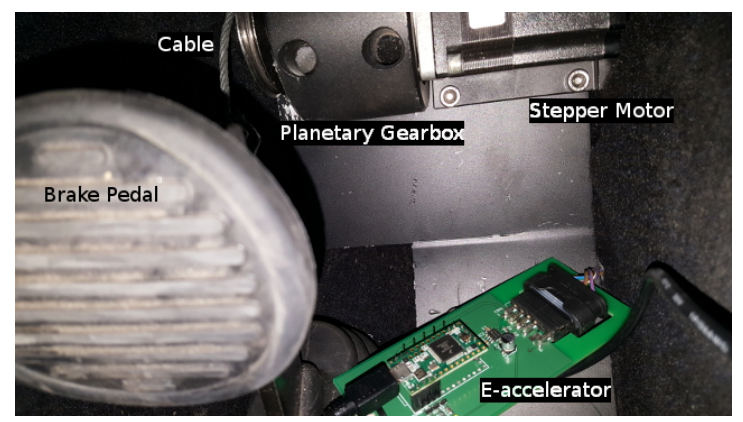

Figure 11: Automatic Accelerator and Brake Actuators [24]

The control input of the proposed NMPC with deadzone-quadratic penalty function is realised by actuating either the accelerator pedal or brake actuator. The accelerator pedal is replaced by an electronic board (E-accelerator) to manipulate the required acceleration and imitates the electric signals generated by the original accelerator pedal of the Smart-ED. The brake actuator is manipulated by an electric stepper motor that is connected to the brake pedal by a planetary gearbox and flexible cable. The automatic brake actuation is designed in a way that preserves the possibility for the driver to brake in emergency cases. Figure 11 shows the configuration of the E-accelerator and brake actuators for the Ext-Eco-CC system.

Figure 12 shows the accelerator transition response of the BEV. Figure 12a shows a full step response from initial value $0 \%$ to $100 \%$ with $130 \mathrm{~ms}$ rise time. Furthermore, the step response from initial value $100 \%$ to $0 \%$ with $130 \mathrm{~ms}$ rise 
(a)
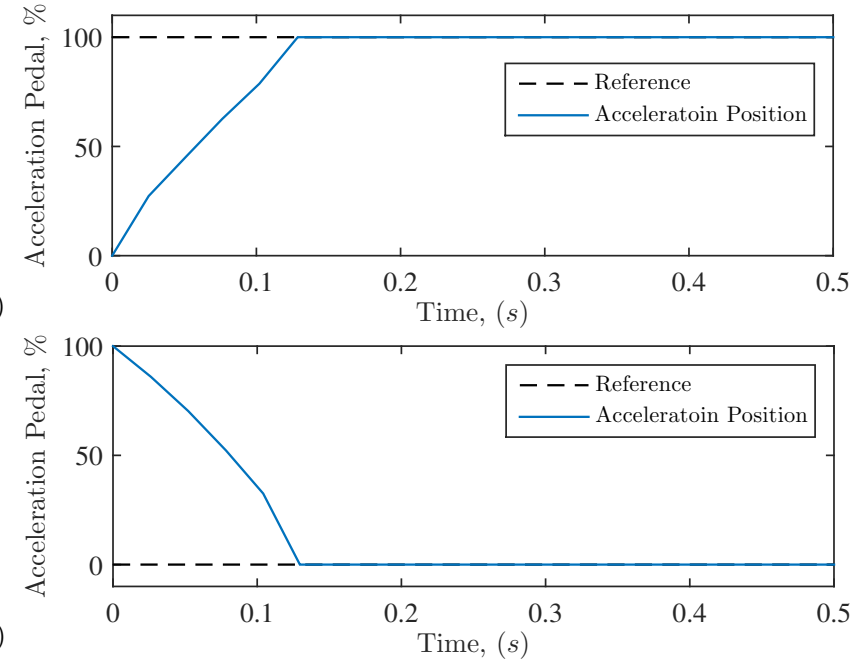

Figure 12: The Smart-ED acceleration transient response (a) $0-100 \%$ Step response, (b) $100-0 \%$ Step response.

time is shown in Figure $12 \mathrm{~b}$. Figure 13 shows the brake transition response of the BEV. It is noteworthy that a two-degree-of-freedom PID controller (2 DOF) as the low level brake actuator controller is implemented. Figure 13a shows a full step response from initial value $0 \%$ to $60 \%$ with $2.7 \mathrm{~s}$ rise time and $0.97 \mathrm{~s}$ Dead-time. Furthermore, the step response from initial value $60 \%$ to $0 \%$ with $0.72 s$ rise time is shown in Figure $13 \mathrm{~b}$. Therefore, it is essential to account for the control input delay in the NMPC. The (51) represents the delay of the control input as follows:

$$
\frac{u_{p}(t)}{d t}=k_{p}\left(u(t)-u_{p}(t)\right)
$$

where $k_{p}$ is the constant the denotes the delay of the control input $u(t)$ [45].

In order to show the performance of the proposed Ext-Eco-CC system, a prediction horizon for the predictive controller is set to $T=15 \mathrm{~s}$, to cover upcoming road geometry, and traffic speed limit zone with $N=30$ discretized steps. The constants in performance index function are set as $Q=\operatorname{diag}[0,2,0]$, and $R=\operatorname{diag}[450]$. Note the weight for energy-consumption is set to zero since the effectiveness of the deadzone-quadratic penalty function in energy efficiency 


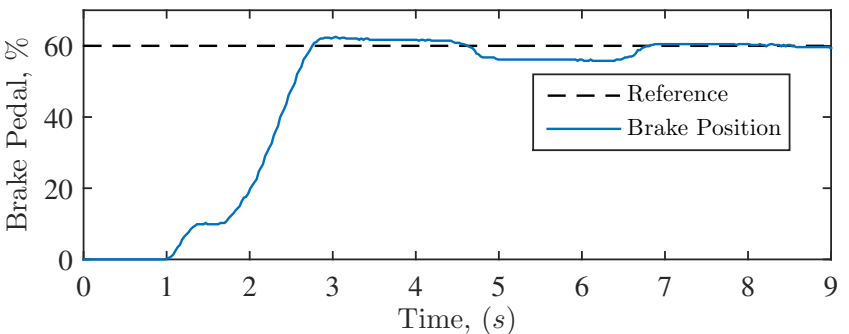

(a)

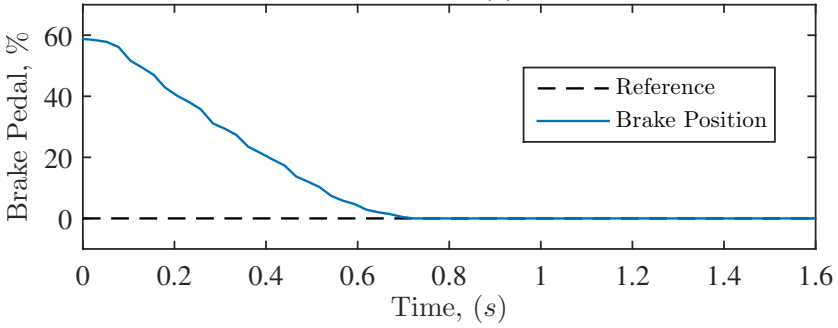

Figure 13: The Smart-ED brake transient response (a) $0-60 \%$ Step response, (b) $60-0 \%$ Step response.

is the main focus in this paper. The reference for the lateral acceleration comfort level is $\Psi_{r e f}=3.7 \mathrm{~m} / \mathrm{s}^{2}$.

Figure 14 shows the field experimental results of the proposed NMPC for the optimal energy management of the BEV on a realistic hilly and curvy road of the test track. In this setup, the BEV is set to cruise at the $v_{r e f}=50 \mathrm{~km} / \mathrm{h}$. Performance of the NMPC (Experiment) is compared with a simulation result (Simulation). Figure 14a shows the BEV velocity profile generated by NMPC for the both Experimental and Simulation. The controllers increase the velocity of the BEV from standstill to reach the desired velocity $\left(v_{r e f}\right)$. However, the velocity decrease as the vehicle approaches the first and second curves which the lateral acceleration constraint become active $(220 \leq s \leq 440)$. After the second curve, the controllers increase the velocity of the BEV to reach the reference value $(550 \leq s \leq 700)$ where it is the only opportunity to reach the desired velocity. By approaching to the third and fourth curves, the velocity of the $\mathrm{BEV}$ has to be reduced to satisfy the lateral acceleration inequality constraint. Figure 14b shows the BEV power consumption profile of the BEV generated by the NMPC for both field experimental and simulation. 

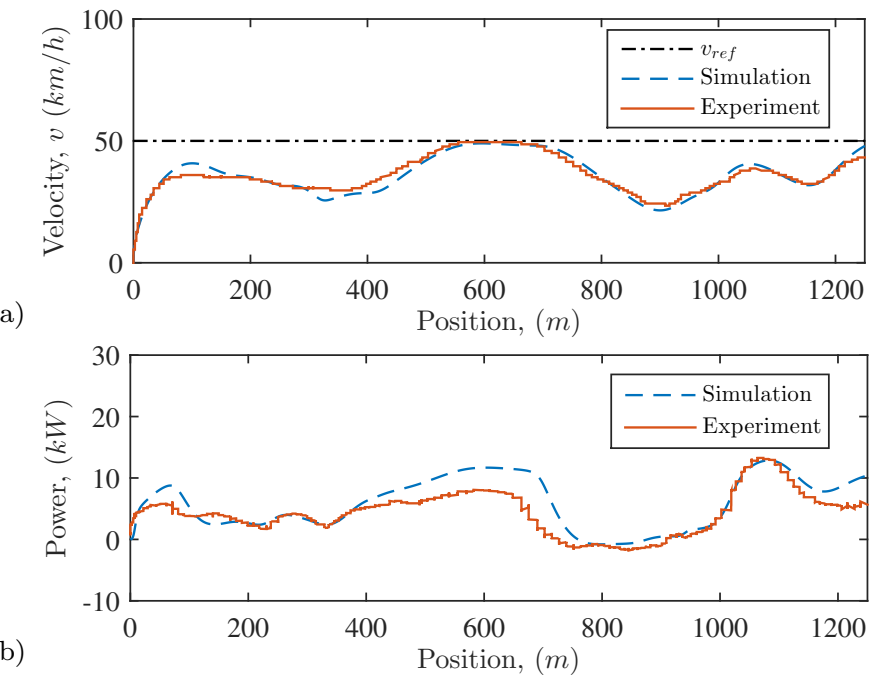

Figure 14: Performance of field experimental NMPC vs. simulation NMPC at $50 \mathrm{~km} / \mathrm{h}$ for (A) Velocity regulation, (B) Power consumption.

We have compared our proposed deadzone-quadratic NMPC (DQ-NMPC) with the conventional NMPC with $\ell_{2}$-norm (C-NMPC) and human driver (HD) in terms of velocity regulation, travel time $(t)$, power consumption profile and total energy-consumption $(e)$. For the sake of fair comparison, all of the tests started from the standstill and the maximum reference velocity value is chosen, $v_{r e f}=100 \mathrm{~km} / \mathrm{h}$ without imposing speed limit zone. The desired reference zone for velocity tracking is chosen as $z=2 \mathrm{~m} / \mathrm{s}$. We have proposed human driver to drive as fast and energy-efficient as possible.

Figure 15a shows the performance of various tests in terms of velocity regulations and total travel time. The DQ-NMPC and NMPC increase the velocity up to reaching the first curve $(220 \leq s \leq 270)$ where the lateral acceleration constraint should be satisfied. As it is shown, the human driver is faster than the controllers. However, during the first and second curves $(320 \leq s \leq 440)$, the controllers and human driver show similar behaviour. Afterwards, the controllers increase over again the velocity up to $68.4 \mathrm{~km} / \mathrm{h}$ with the DQ-NMPC setup and $70.2 \mathrm{~km} / \mathrm{h}$ with the NMPC configuration and slowing down at the 


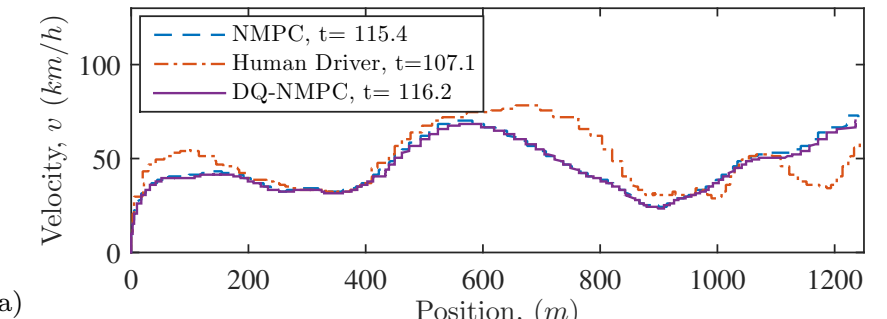

(a)

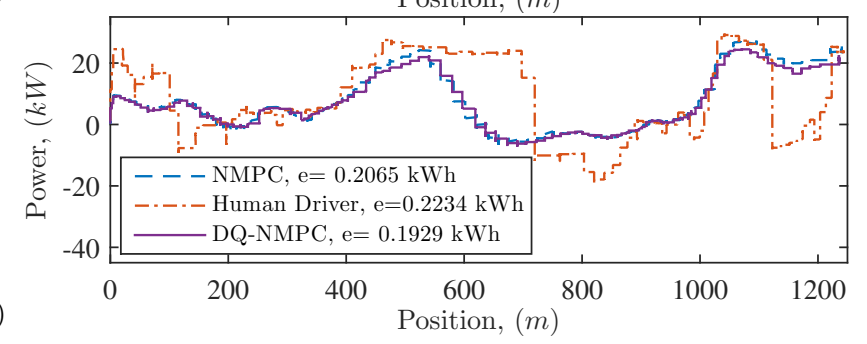

Figure 15: Experimental results of DQ-NMPC in comparison with NMPC and Human Driver for (a) Velocity regulation, and (b) Power consumption profile.

point the third curve $(860 \leq s \leq 930)$ are in their prediction horizon. This leads to the beginning of slowing down predictively to satisfy the upcoming constraints in an energy-efficient way. The human driver hits the maximum $78.3 \mathrm{~km} / \mathrm{h}$ and shows late but sharper velocity reduction which may not be an energy-efficient technique. Finally, the controllers keep the velocity during the fourth curve $(930 \leq s \leq 1045)$ and speed up once more to reach the starting point on the test track. Thus, the presented result shows that the maximum reference velocity is not reachable, however, the reference velocity for less than $v_{\text {ref }}=80 \mathrm{~km} / \mathrm{h}$ is reachable on the experimental tests carried on the test track. Figure $15 \mathrm{~b}$ shows the power consumption profile and total used energy. Note that negative power consumption refers to energy recovery mechanism. The DQ-NMPC and NMPC show relatively similar behaviour, however, the DQNMPC could avoid peak velocity and power consumption in comparison to the NMPC for higher velocity. These situations provide the opportunities to save energy consumption of the BEV.

Figure $16 \mathrm{a}$ and Figure $16 \mathrm{~b}$ show the velocity and power consumption normalised histogram information. The proposed DQ-NMPC benefits from an im- 


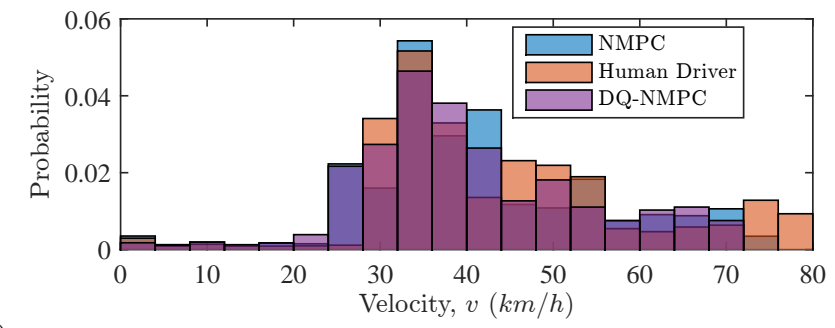

(a)

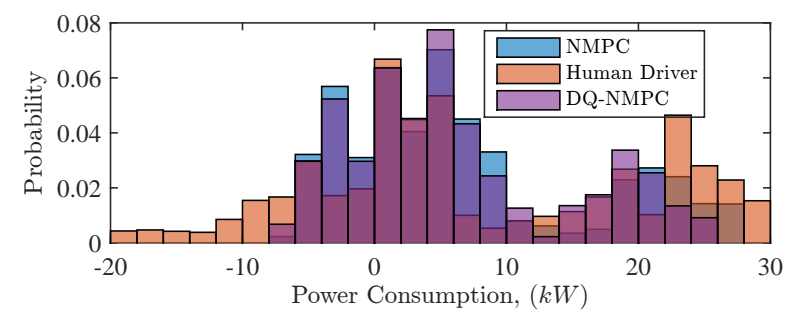

(b)

Figure 16: Experimental performance distribution of DQ-NMPC in comparison with NMPC and the Human Driver for (A) Velocity and (B) Power consumption.

proved penalty function which leads to a denser velocity and power consumption distribution in comparison to the NMPC and human driver. Based on achieved results, it is shown that the set-point value is not reachable on the test track by the controllers or the human driver. The DQ-NMPC leads to more steady velocity profile and consequently the better drive comfort with relatively small increased travel time. The total energy consumption of DQ-NMPC is $+13.65 \%$ more energy efficient in comparison to the human driver and $+6.58 \%$ more energy efficient in comparison to the NMPC. In other words, for longer trips with more hilly and curvy roads, the proposed method has higher potential to be more energy-efficient. It is noteworthy that the OCP average calculation time for the DQ-NMPC is $2.35 \mathrm{~ms}$ which indicates the real-time capability of the 475 proposed controller. Figure 17 shows the probability distribution of the accelerator and brake actuators. Figure 17a shows that the DQ-NMPC has relatively denser distribution in comparison to the NMPC and the human driver where 


\begin{tabular}{c|c|c|c} 
& Human Driver & NMPC & DQ-NMPC \\
\hline Eco. (\%) & - & $+7.56 \%$ & $+13.65 \%$
\end{tabular}

Table 1: Energy consumption improvement of the Smart-ED

Figure 17b demonstrates a similar brake pedal distribution for the DQ-NMPC, the NMPC, and the human driver.

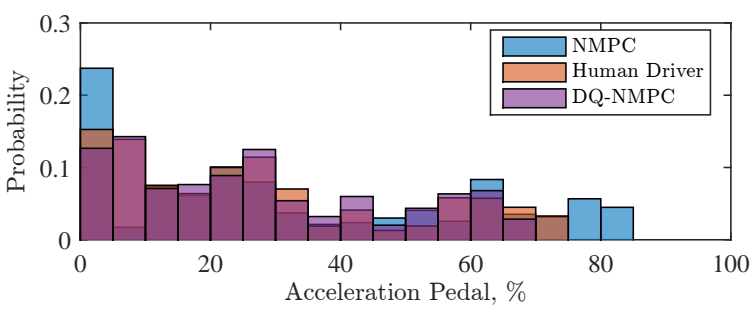

(a)

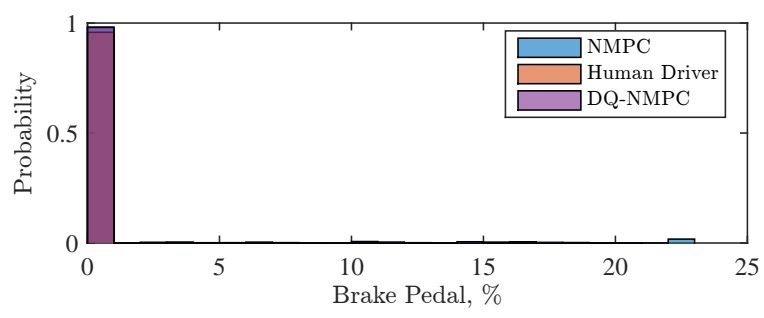

Figure 17: Experimental performance distribution of DQ-NMPC in comparison with NMPC and the Human Driver for (A) Acceleration pedal and (B) Brake pedal.

The NMPC formulation, numerical simulation, and field experimental results were presented in this section. The performance of the Ext-Eco-CC system was evaluated in terms of safety and energy efficiency. This paper extends the NMPC formulation of the Ext-Eco-CC system introduced in 21 with nonlinear FB complementary function aimed to enhance the inequality constraint handling method. In addition, the Ext-Eco-CC system introduced in [21] and 24] were rigorously evaluated with numerical simulation and field experimental results in this paper. An overall energy consumption is given in Table 1 to conclude this section. 


\section{Conclusion and Future Research}

490

A semi-autonomous ecological driver assistance system was developed to regulate the velocity in an energy-efficient manner for the electric vehicles. This system aimed to address the shortcoming of limited cruising range of the battery electric vehicles. A real-time nonlinear receding horizon optimal controller with approximate deadzone-quadratic penalty function was proposed to plan online the cost-effective velocity profile based on the vehicle dynamics, its energy consumption characteristic map, and road geometry with traffic sign information. Although a relatively complex problem was formulated, the computational time is found to be short. The inequality constraints of the predictive controller were handled based on the proposed soft nonlinear complementary function to enhance the minimum principle method. The limited cruising range of the electric vehicles was improved by the assessed low penalty value on set-point tracking zone and ecological driving techniques. Various tests on a semi-autonomous electric vehicle in terms of real-time states regulation and constraints fulfilment were carried out. The effectiveness of the proposed method was demonstrated by the achieved numerical simulation and field experimental results. Further practical experiments will be conducted including extending the functionalities of the semi-autonomous ecological driving.

\section{References}

[1] A. Eskandarian (Ed.), Handbook of Intelligent Vehicles, Vol. 2, Springer London, London, 2012. doi:10.1007/978-0-85729-085-4.

[2] S. Manzetti, F. Mariasiu, Electric vehicle battery technologies: From present state to future systems, Renewable and Sustainable Energy Reviews 51 (2015) 1004-1012. doi:10.1016/j.rser.2015.07.010.

[3] B. Sarlioglu, C. T. Morris, D. Han, S. Li, Driving Toward Accessibility: A Review of Technological Improvements for Electric Machines, Power 
Electronics, and Batteries for Electric and Hybrid Vehicles, IEEE Indus-

try Applications Magazine 23 (1) (2017) 14-25. doi:10.1109/MIAS.2016. 2600739

[4] H. Rouzikhah, M. King, A. Rakotonirainy, Examining the effects of an ecodriving message on driver distraction, Accident Analysis \& Prevention 50 (2013) 975-983. doi:10.1016/j .aap.2012.07.024

[5] A. Sciarretta, G. De Nunzio, L. L. Ojeda, Optimal Ecodriving Control: Energy-Efficient Driving of Road Vehicles as an Optimal Control Problem, IEEE Control Systems 35 (5) (2015) 71-90. doi:10.1109/MCS. 2015. 2449688

[6] I. K. Luigi Del Re, Frank Allgöwer, Luigi Glielmo, Carlos Guardiola, Automotive Model Predictive Control, Vol. 402 of Lecture Notes in Control and Information Sciences, Springer London, London, 2010. doi: 10.1007/978-1-84996-071-7

[7] I. Koryakovskiy, M. Kudruss, R. Babuka, W. Caarls, C. Kirches, K. Mom-

a baur, J. P. Schlder, H. Vallery, Benchmarking model-free and model-based optimal control, Robotics and Autonomous Systems 92 (2017) 81 - 90. doi:https://doi.org/10.1016/j.robot.2017.02.006. URL http://www.sciencedirect.com/science/article/pii/ S0921889016301592

[8] M. R. Azizi, J. Keighobadi, Point stabilization of nonholonomic spherical mobile robot using nonlinear model predictive control, Robotics and $\mathrm{Au}-$ tonomous Systems 98 (2017) 347-359. doi:10.1016/j.robot.2017.09. 015 .

${ }_{540}^{\text {口 }}$ [9] Y. Huang, H. Wang, A. Khajepour, H. He, J. Ji, Model predictive control power management strategies for HEVs: A review, Journal of Power Sources 341 (2017) 91-106. doi:10.1016/j.jpowsour.2016.11.106. URL http://linkinghub.elsevier.com/retrieve/pii/S0378775316316731 
[10] S. Park, H. Rakha, K. Ahn, K. Moran, Predictive eco-cruise control:

[11] B. Saerens, H. Rakha, M. Diehl, E. Van den Bulck, A methodology for assessing eco-cruise control for passenger vehicles, Transportation Research 550 Part D: Transport and Environment 19 (2013) 20-27. doi:10.1016/j.trd. 2012.12 .001

[12] M. a. S. Kamal, M. Mukai, J. Murata, T. Kawabe, Ecological Vehicle Control on Roads With Up-Down Slopes, IEEE Transactions on Intelligent

घ Transportation Systems 12 (3) (2011) 783-794. doi:10.1109/TITS.2011. 2112648

[13] W. Dib, A. Chasse, P. Moulin, A. Sciarretta, G. Corde, Optimal energy management for an electric vehicle in eco-driving applications, Control En-

ㅁ gineering Practice 29 (2014) 299-307. doi:10.1016/j.conengprac. 2014. 01.005

[14] T. Schwickart, H. Voos, J.-R. Hadji-Minaglou, M. Darouach, A novel model-predictive cruise controller for electric vehicles and energy-efficient driving, in: 2014 IEEE/ASME International Conference on Advanced In-

a telligent Mechatronics, no. Section IV, IEEE, 2014, pp. 1067-1072. doi: 10.1109/AIM.2014.6878222

565

[15] T. Schwickart, H. Voos, J.-R. Hadji-Minaglou, M. Darouach, A. Rosich, Design and simulation of a real-time implementable energy-efficient modelpredictive cruise controller for electric vehicles, Journal of the Franklin

․ Institute 352 (2) (2015) 603-625. doi:10.1016/j.jfranklin.2014.07. 001.

570 [16] B. Alrifaee, Y. Liu, D. Abel, ECO-cruise control using economic model predictive control, in: 2015 IEEE Conference on Control Applications (CCA), IEEE, 2015, pp. 1933-1938. doi:10.1109/CCA.2015.7320892. 
[17] T. Schwickart, H. Voos, J.-R. Hadji-Minaglou, M. Darouach, A Fast ModelPredictive Speed Controller for Minimised Charge Consumption of Electric Vehicles, Asian Journal of Control 18 (1) (2016) 133-149. doi:10.1002/ asjc.1251.

[18] M. Batra, J. McPhee, N. L. Azad, Anti-jerk model predictive cruise control for connected electric vehicles with changing road conditions, in: 2017 11th Asian Control Conference (ASCC), 2017, pp. 49-54. doi:10.1109/ASCC. 2017.8287101

[19] L. Grüne, J. Pannek, Nonlinear Model Predictive Control, Vol. 1 of Communications and Control Engineering, Springer London, London, 2011. doi:10.1007/978-0-85729-501-9.

[20] T. Schwickart, H. Voos, J.-R. Hadji-Minaglou, M. Darouach, An efficient nonlinear model-predictive eco-cruise control for electric vehicles, in: 2013 11th IEEE International Conference on Industrial Informatics (INDIN), IEEE, 2013, pp. 311-316. doi:10.1109/INDIN.2013.6622901.

[21] S. A. Sajadi-alamdari, H. Voos, M. Darouach, Nonlinear Model Predictive Extended Eco-Cruise Control for Battery Electric Vehicles, in: 24th Mediterranean Conference on Control and Automation (MED), IEEE, Athens, 2016, pp. 467-472. doi:10.1109/MED.2016.7535929.

[22] S. Boyd, L. Vandenberghe, Convex Optimization, Cambridge University Press, 2004. doi:10.1017/CB09780511804441.

[23] S. Gros, M. Zanon, Penalty Functions for Handling Large Deviation of Quadrature States in NMPC, IEEE Transactions on Automatic Control 9286 (c) (2017) 1-1. doi:10.1109/TAC.2017.2649043.

[24] S. A. Sajadi-Alamdari, H. Voos, M. Darouach, Deadzone-quadratic penalty function for predictive extended cruise control with experimental validation, in: A. Ollero, A. Sanfeliu, L. Montano, N. Lau, C. Cardeira (Eds.), ROBOT 2017: Third Iberian Robotics Conference, Springer International 
Publishing, Cham, 2018, pp. 446-459. doi:10.1007/978-3-319-70836-2_ 37.

[25] M. Ehsani, Y. Gao, A. Emadi, Modern Electric, Hybrid Electric, and Fuel Cell Vehicles: Fundamentals, Theory, and Design, 2nd Edition, CRC Press, 2009.

[26] C. Fiori, K. Ahn, H. A. Rakha, Power-based electric vehicle energy consumption model: Model development and validation, Applied Energy 168 (2016) 257-268. doi:10.1016/j . apenergy .2016.01.097

URL http://linkinghub.elsevier.com/retrieve/pii/S030626191630085X

[27] F. Borrelli, A. Bemporad, M. Morari, Predictive control for linear and hybrid systems, 2014.

URL http://www.mpc.berkeley.edu/mpc-course-material

[28] M. Huang, H. Nakada, K. Butts, I. Kolmanovsky, Nonlinear Model Predictive Control of a Diesel Engine Air Path: A Comparison of Constraint Handling and Computational Strategies, IFAC-PapersOnLine 48 (23) (2015) 372-379. doi:10.1016/j.ifacol.2015.11.308.

[29] T. Ohtsuka, A continuation/GMRES method for fast computation of nonlinear receding horizon control, Automatica 40 (4) (2004) 563-574. doi:10.1016/j.automatica.2003.11.005

[30] M. Diehl, H. J. Ferreau, N. Haverbeke, Efficient Numerical Methods for Nonlinear MPC and Moving Horizon Estimation, in: Workshop on Asn sessment and Future Directions of NMPC, 2009, pp. 391-417. doi: 10.1007/978-3-642-01094-1_32.

[31] T. Ohtsuka, K. Ozaki, Practical Issues in Nonlinear Model Predictive Control: Real-Time Optimization and Systematic Tuning, 2009, pp. 447-460. doi:10.1007/978-3-642-01094-1_35 
[32] C. T. Kelley, Iterative Methods for Linear and Nonlinear Equations, Vol. 16,

口 Society for Industrial and Applied Mathematics, 1995. doi:10.1137/1. 9781611970944

[33] Y. Shimizu, T. Ohtsuka, M. Diehl, A real-time algorithm for nonlinear receding horizon control using multiple shooting and continuation/krylov method, International Journal of Robust and Nonlinear Control 19 (8) (2009) 919-936. doi:10.1002/rnc.1363.

[34] K. Graichen, A. Kugi, N. Petit, F. Chaplais, Handling constraints in optimal control with saturation functions and system extension, Systems \& Control Letters 59 (11) (2010) 671-679. doi:10.1016/j.sysconle.2010. 08.003

[35] A. Fischer, A special newton-type optimization method, Optimization 24 (3-4) (1992) 269-284. doi:10.1080/02331939208843795

[36] A. F. Izmailov, M. V. Solodov, Newton-Type Methods for Optimization and Variational Problems, Springer Series in Operations Research and Financial Engineering, Springer International Publishing, Cham, 2014. doi : 10.1007/978-3-319-04247-3.

[37] C. Kanzow, H. Pieper, Jacobian Smoothing Methods for Nonlinear Complementarity Problems, SIAM Journal on Optimization 9 (2) (1999) 342-373. doi:10.1137/S1052623497328781.

[38] M. Fukushima, Z.-Q. Luo, J.-S. Pang, A Globally Convergent Sequential Quadratic Programming Algorithm for Mathematical Programs with Linear Complementarity Constraints, Computational Optimization and Applications 10 (1) (1998) 5-34. doi:10.1023/A:1018359900133

[39] H. Jiang, D. Ralph, Smooth SQP Methods for Mathematical Programs with Nonlinear Complementarity Constraints, SIAM Journal on Optimization 10 (3) (2000) 779-808. doi:10.1137/S1052623497332329. 
[40] J. Tang, L. Dong, J. Zhou, L. Fang, A smoothing Newton method for nonlinear complementarity problems, Computational and Applied Mathematics 32 (1) (2013) 107-118. doi:10.1007/s40314-013-0015-9.

[41] J. M. Maciejowski, Predictive Control: With Constraints, no. Pearson Education, Prentice Hall, 2002.

[42] Y. LeCun, Y. Bengio, G. Hinton, Deep learning, Nature 521 (7553) (2015) $436-444$.

[43] C. Dugas, Y. Bengio, F. Bélisle, C. Nadeau, R. Garcia, Incorporating Second-order Functional Knowledge for Better Option Pricing, in: Proceedings of the 13th International Conference on Neural Information Processing Systems, NIPS'00, MIT Press, Cambridge, MA, USA, 2000, pp. 451-457.

[44] Centre de Formation pour Conducteurs, Colmar-Berg, Luxembourg. URL http://www.cfc.lu/

[45] D. Yamaguchi, M. Kamal, M. Mukai, T. Kawabe, Model Predictive Control for Automobile Ecological Driving Using Traffic Signal Information, 670 J Journal of System Design and Dynamics 6 (3) (2012) 297-309. doi: $10.1299 /$ jsdd.6.297 